\title{
Effective use of image-enhanced endoscopy and endoscopic submucosal dissection for multiple flat non-polypoid colorectal neoplasms
}

Non-polypoid colorectal neoplasms are the precursors of post-colonoscopy colorectal cancers (PCCRCs) [1], but can easily be overlooked because of their appearance. Therefore, early detection and treatment of laterally spreading tumors, non-granular type (LST-NGs) and 0-IIb lesions are important for preventing PCCRC. We report the case of a patient with multiple flat non-polypoid colorectal neoplasms, among which a 0 -IIb (LST-NG) lesion was appropriately diagnosed with image-enhanced endoscopy and treated with endoscopic submucosal dissection (ESD) [2].

A 58-year-old woman whose mother and uncle had a history of CRC had a positive fecal occult blood test result. Multiple flat non-polypoid colorectal neoplasms with diverse morphologies, including a type 2 lesion, were detected by subsequent colonoscopy ( $\triangleright$ Fig. 1). Right hemicolectomy was scheduled for the type 2 lesion in the transverse colon. Another colonoscopy was performed for close examination before surgery.

A 35-mm 0-IIb (LST-NG) lesion, which had an unclear margin on white-light imaging, was detected in the descending coIon. The lesion was clearly visualized using narrow-band imaging (NBI) and indigo carmine dye. Magnifying NBI showed a Japan NBI Expert Team (JNET) type 2B pattern [3], and crystal violet staining indicated a type $V_{ı}$ (non-invasive) pit pattern ( $\boldsymbol{F i g . 2}$ ). The preoperative diagnosis was intramucosal or submucosal superficial invasive cancer $(<1000 \mu \mathrm{m})$, and the lesion was located outside the right hemicolectomy area. It was therefore resected via ESD, and curative resection was achieved (\$ Fig.3; - Video 1). The tumor was intact for the two mismatch repair proteins (MSH6, PMS2) by immunohistochemical staining. No germline pathogenic variant was found in the cancer predisposition genes, including MLH1, MSH2, MSH6, PMS2, PTEN, and TP53 by multigene panel test-

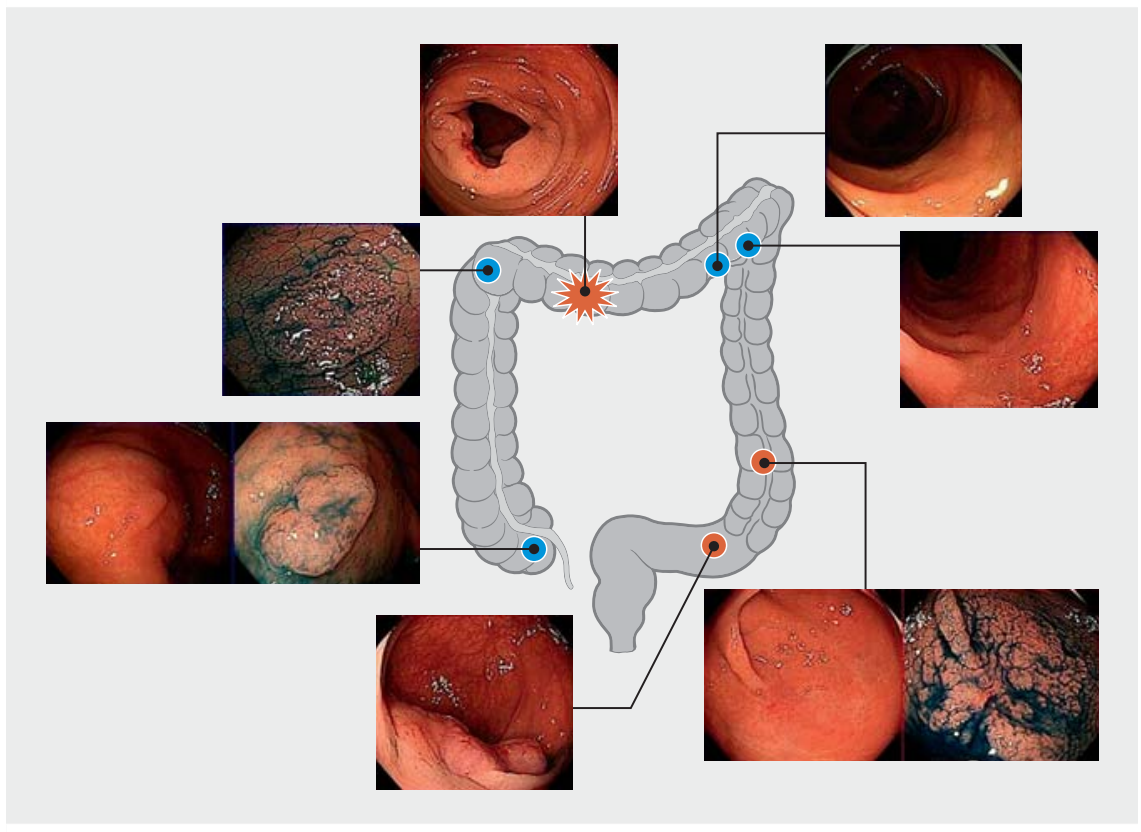

- Fig. 1 Endoscopic views of the multiple colorectal neoplasms, with a schematic marking where they were identified, showing a $10-\mathrm{mm} 0$-Is lesion in the cecum; a $12-\mathrm{mm}$ lesion at the hepatic flexure; a $40-\mathrm{mm}$ type 2 lesion in the transverse colon; a 15-mm 0-Ila lesion in the transverse colon; a 0-IIb lesion in the transverse colon; a 40-mm 0-Is + Ila lesion in the sigmoid colon; and a 35-mm 0-IIb laterally spreading tumor, non-granular type, in the descending colon.
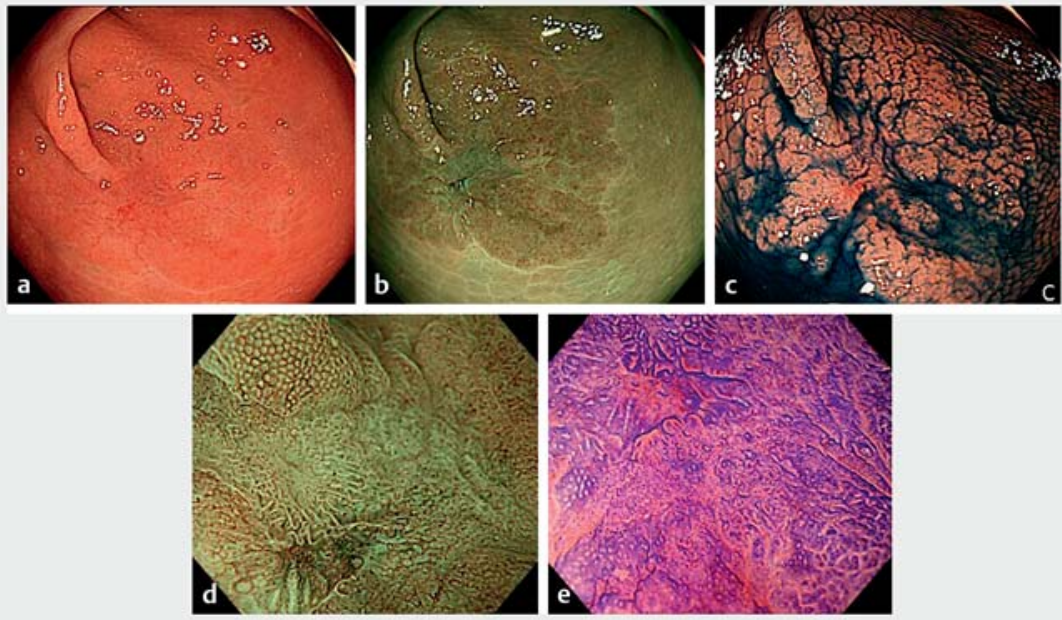

Fig. 2 Endoscopic images showing: a a 35-mm 0-Ilb laterally spreading tumor, non-granular type, on white-light endoscopy; b, $\mathbf{c}$ the tumor margin demonstrated with: $\mathbf{b}$ narrow-band imaging (NBI); $\mathbf{c}$ indigo carmine dye; $\mathbf{d}$ an irregular distribution of vessels and a visible irregular surface pattern on magnifying NBI; e an uneven and irregular pit pattern without territoriality on crystal violet staining. 


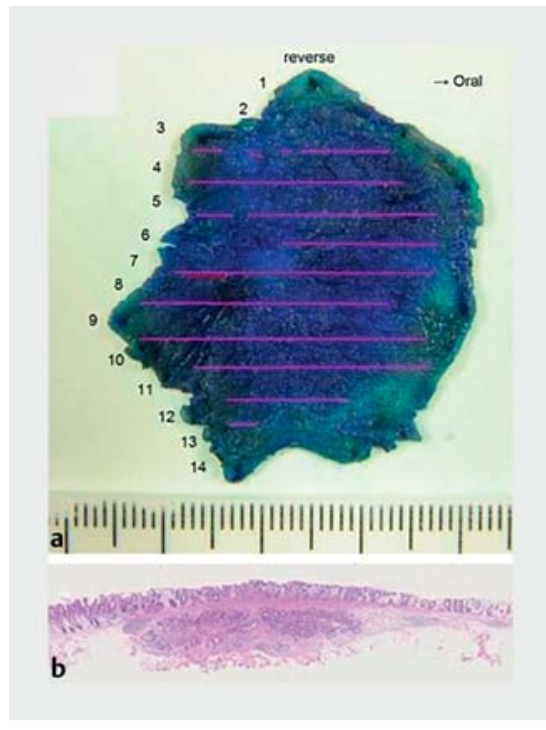

- Fig. 3 Histopathological appearance of the resected lesion showing: a tumorfree horizontal and vertical margins and no evidence of lymphovascular invasion (pink lines represent adenocarcinoma in the mucosal layer; the red line represents adenocarcinoma in the submucosal layer); b the microscopic appearance with a depth of $750 \mu \mathrm{m}$ indicating invasion into the submucosal layer without lymphovascular invasion, consistent with a curative resection having been achieved.

ing using peripheral blood. After the ESD had been completed, the type 2 lesion was surgically treated.

Appropriate diagnosis and treatment of non-polypoid colorectal neoplasms are crucial for the prevention of postoperative metachronous CRC and PCCRC.

Endoscopy_UCTN_Code_CCL_1AD_2AC

\section{Competing interests}

The authors declare that they have no conflict of interest.

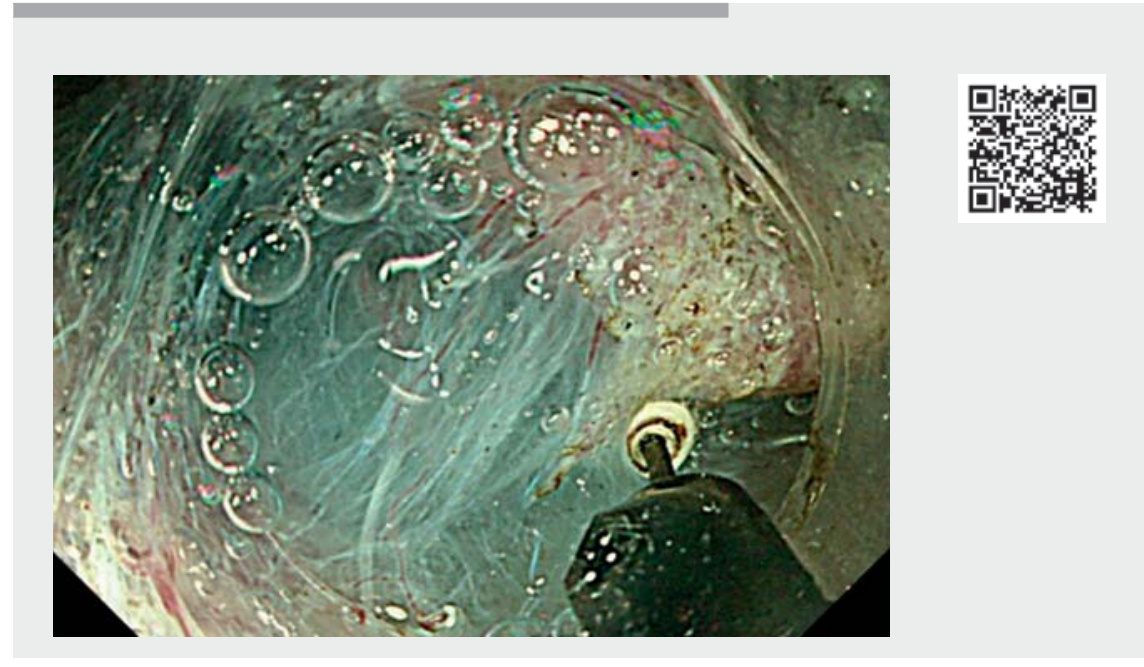

$\checkmark$ Video 1 Image-enhanced endoscopy and endoscopic submucosal dissection of a 0-Ilb lesion in the descending colon.

The authors

Takeshi Uozumi ${ }^{1}$, Yutaka Saito ${ }^{1}$, Naoya Toyoshima', Hiroyuki Takamaru' ${ }^{1}$, Masau Sekiguchi ${ }^{1,2}$, Masayoshi Yamada ${ }^{1}$, Seiichiro Abe ${ }^{1 \odot}$

1 Endoscopy Division, National Cancer Center Hospital, Chuo-ku, Tokyo, Japan

2 Cancer Screening Center, National Cancer Center Hospital, Chuo-ku, Tokyo, Japan

\section{Corresponding author}

\section{Yutaka Saito, MD, PhD}

Endoscopy Division, National Cancer Center Hospital, 5-1-1, Tsukiji, Chuo-ku, Tokyo 104-0045, Japan ytsaito@ncc.go.jp

\section{References}

[1] Matsuda T, Fujii T, Sano Y et al. Randomised comparison of postpolypectomy surveillance intervals following a two-round baseline colonoscopy: the Japan Polyp Study Workgroup. Gut 2020; 70: 1469-1478

[2] Saito Y, Abe S, Inoue H et al. How to perform a high-quality endoscopic submucosal dissection. Gastroenterology 2021; 161: 405410

[3] Sano Y, Tanaka S, Kudo SE et al. Narrowband imaging (NBI) magnifying endoscopic classification of colorectal tumors proposed by the Japan NBI Expert Team. Dig Endosc 2016; 28: 526-533
Bibliography

Endoscopy 2022; 54: E684-E685

DOI 10.1055/a-1753-9294

ISSN 0013-726X

published online 18.2.2022

(c) 2022. Thieme. All rights reserved.

Georg Thieme Verlag KG, Rüdigerstraße 14

70469 Stuttgart, Germany

\section{CORRECTION}

Effective use of image-enhanced endoscopy and endoscopic submucosal dissection for multiple flat non-polypoid colorectal neoplasms

Uozumi T, Saito $\mathrm{Y}$, Toyoshima $\mathrm{N}$ et al. Effective use of image-enhanced endoscopy and endoscopic submucosal dissection for multiple flat nonpolypoid colorectal neoplasms. Endoscopy 2022, doi:10.1055/a-17539294

In the above-mentioned article, the classification of tumors has been corrected to $0-\mathrm{IIb}$. This was corrected in the online version on March 24, 2022. 